\section{Efecto de las variantes de CYP2C9 sobre la dosis de losartán en hipertensos chilenos}

\author{
CRISTIAN PEDREROS-ROSALES ${ }^{1,2}$, ROBERTO JALIL MILAD ${ }^{1}$, \\ MARCELA LAGOS LUCERO ${ }^{3,4}$, SANDRA SOLARI GAJARDO $^{3}$
}

\section{Association between cytochrome P4502C9 polymorphisms and losartan dosing in hypertensive patients}

Background: Losartan is widely used in many clinicals settings. Its dosage is related to the genetic characteristics of CYP2C9 enzymatic activity, which metabolizes losartan to its active form E-3174, responsible for the antihypertensive effect. Aims: To identify the frequency of allelic variants CYP2C9*2 and $\mathrm{CYP}_{2} \mathrm{C9}^{*} 3$ in hypertensive patients and to compare genotypes with a healthy Chilean population. To relate polymorphisms with the losartan dosing to obtain an optimal blood pressure. Material and Methods: We studied 30 patients with controlled essential hypertension using losartan with normal liver function, and 202 healthy people. Peripheral blood DNA genotyping was performed by polymerase chain reaction to identify the polymorphisms. Allelic and genotypic frequencies were compared. Results: In hypertensive patients, allelic frequencies were $0.85\left(C Y P 2 C 9^{*} 1\right), 0.05\left(C Y P 2 C 9^{\star} 2\right)$ and $0.1\left(C Y P 2 C 9^{*} 3\right)$. Genotypic frequencies were $73.3 \%\left(C Y P 2 C 9^{*} 1{ }^{*} 1\right), 6.7 \%\left(C Y P 2 C 9^{\star} 1{ }^{*} 2\right)$, $16.7 \%\left(C Y P 2 C 9^{*} 1 /^{*} 3\right)$ and $3.3 \%\left(C Y P 2 C 9^{*} 2 / 3\right)$; observing a significantly higher frequency of the allele $C Y P 2 C 9^{*} 3(p=0.041)$ and $C Y P 2 C 9^{*} 1{ }^{*} 3$ genotype $(p=0.04)$. A non-significant tendency to need a larger dose of losartan was observed with the CYP2C9 3 allele, with an odds ratio (OR) of $1.46(95 \%$ confidence intervals (CI) 0.01-18.64). The same tendency was observed with the need to use losartan twice a day, obtaining an OR of 5.88 (CI 0.54 -62.14). Conclusions: There could be a relationship between the presence of CYP2C9 polymorphisms and the pathogenesis of hypertension. The presence of CYP2C9*3 is associated with the need for higher doses of losartan, possibly due to a decrease in the conversion of losartan to E-3174.

(Rev Med Chile 2019; 147: 1527-1534)

Key words: Cytochrome P-450 CYP2C9; Genotype; Losartan; Polimorphism, Genetic.
'Departamento de Nefrología Facultad de Medicina, Pontificia Universidad Católica de Chile. Santiago, Chile.

${ }^{2}$ Departamento de Medicina Interna, Facultad de Medicina, Universidad de Concepción.

Concepción, Chile.

${ }^{3}$ Departamento de Laboratorios Clínicos, Facultad de Medicina,

Pontificia Universidad Católica de

Chile. Santiago, Chile.

"Laboratorio de Biología Molecular y Citogenética, Facultad de Medicina, Pontificia Universidad Católica de Chile. Santiago, Chile.

Trabajo no recibió financiamiento. Los autores declaran no tener conflictos de interés.

Recibido el 7 de junio de 2019, aceptado el 9 de octubre de 2019.

Correspondencia a:

Dr. Cristian Pedreros Rosales Facultad de Medicina Universidad de Concepción. Los Sauces 58, Las Higueras. Talcahuano, Chile. cpedreros@me.com
I

osartán fue descrito para su uso humano por primera vez en 1990, como el primer antagonista no-péptido, altamente selectivo del receptor 1 (AT1) de angiotensina $\mathrm{II}^{1} \mathrm{y}$ desde entonces es ampliamente usado en el tratamiento de la hipertensión arterial y la insuficiencia cardía$\mathrm{Ca}^{2,3}$. Pese a lo anterior, hasta hoy no hay consenso en relación a su posología, siendo recomendado usar "una o dos veces al día" 3,4 , lo que posiblemente tiene que ver, entre otras condiciones, con diferencias genéticas en la actividad enzimática del metabolismo de la droga que conduce a una variabilidad muy amplia interindividual en la respuesta al fármaco, resultando en una eficacia alterada ${ }^{5}$. 
Estudios in vitro ${ }^{6,7}$ e in vivo ${ }^{8,9}$ demostraron que losartán se metaboliza por CYP450, particularmente por su isoenzima CYP2C $9^{10}$. Tras la ingesta oral de una dosis, losartán es rápidamente absorbido y luego metabolizado por CYP2C9 lo que da origen a la formación de siete metabolitos ${ }^{11}$. Los productos monohidroxilados y los conjugados de ácido glucurónidos son mucho menos activos que losartán, mientras que el $14 \%$ aproximadamente se transforma en ácido 5-carboxílico, también llamado E-3174, ${ }^{5,10,11}$ y que es 10 veces más potente que el losartán como antagonista del receptor $\mathrm{AT}^{12}{ }^{12}$. Después de la ingestión, se obtienen valores plasmáticos máximos de losartán a la hora y a las $3 \mathrm{~h}$ en el caso de E-3174 y se ha observado que el área bajo la curva de concentración plasmática (AUC) es 4 veces mayor que losartán, siendo E-3174 finalmente el responsable del efecto antihipertensivo ${ }^{13}$.

En humanos, la subfamilia CYP2C corresponde aproximadamente a $18 \%$ de las proteínas CYP que se encuentran en los microsomas hepáticos, siendo responsable de catalizar $20 \%$ de las reacciones metabólicas de distintas drogas ${ }^{7}$. CYP2C9 es la principal isoenzima involucrada en la oxidación de diversos fármacos de uso habitual como son warfarina ${ }^{14}$, fenitoína ${ }^{15}$, varios anti-inflamatorios no esteroidales ${ }^{16}$, tolbutamida ${ }^{17}$ y de algunos antihipertensivos, como irbersartán ${ }^{18}$ y losartán ${ }^{2}$. Por otra parte, se ha identificado la presencia de CYP2C9 en tejidos cardiovasculares y se sugiere que esta enzima tendría un rol fisiológicamente relevante en la producción de eicosanoides vasoactivos, lo cual pudiera ser importante en la modulación del tono vascular, especialmente porque CYP2C9 es la epoxigenasa principal de CYP450 en aorta y arterias coronarias ${ }^{19}$ y se sabe que su actividad metaboliza ácido araquidónico a ácido eicosatrienoico (EET), el cual es un potente vasodilatador ${ }^{20} \mathrm{y}$ además tendría un rol protector en la homeostasis del endotelio ${ }^{21}$.

Tal como ocurre con otras isoformas de CYP, el gen CYP2C9 es altamente polimórfico y sus variantes tienen repercusiones clínicas que pueden resultar en toxicidad o alteración en la eficacia de algunos fármacos ${ }^{7}$. El alelo $C Y P 2 C 9^{*} 1$, es considerado normal o "wild-type"22, mientras que CYP2C9² (Arg144Cys) y CYP2C9³ (Ile359Leu) son las variantes más frecuentes entre la población caucásica $^{7}$. Estos dos polimorfismos se asocian a una disminución en la transformación de losar- tán a E-3174 tanto in vitro ${ }^{10}$, como in vivo ${ }^{12,23-26}$, observándose una baja actividad enzimática en la variante alélica $C Y P 2 C 9^{\star} 2$ y aún menor en el caso de $C Y P 2 C 9^{*} 3$. Estos efectos son más acentuados en el caso de ser homocigoto para estas variantes ${ }^{7}$, pudiendo llegar a una conversión de losartán a E-3174 menor de $1 \%$ en el caso de presentarse el genotipo $C Y P 2 C 9^{*} 3 /^{*} 3$, a quienes se les llama "metabolizadores lentos"11.

En la literatura internacional, hay varios estudios que relacionan el efecto de las variantes de CYP2C9 en el metabolismo de losartán, pero no existe evidencia sólida aún que permita relacionar los diversos genotipos con su uso clínico. Existen muy pocos estudios en población hispanoamericana y dado que losartán se usa ampliamente en nuestro país, nos parece importante conocer la relación entre los polimorfismos de CYP2C9 y el uso de losartán considerando la falta de información en nuestro grupo étnico. Dicha información puede ser relevante en varios aspectos. Entre otras, permitiría ajustar el tratamiento para lograr un buen control tensional durante las $24 \mathrm{~h}$ del día, lo que incluye mantener el descenso nocturno de la presión arterial (DIP) por su conocido beneficio sobre el riesgo cardiovascular y daño de órgano blanco ${ }^{27}$. Además de lograr este objetivo clínico, se agrega el beneficio económico que implica usar el fármaco de acuerdo con la posología correcta.

Este estudio tiene como objetivos identificar la frecuencia de las variantes alélicas $C Y P 2 C 9^{*} 2$ y CYP2C9 ${ }^{*} 3$ en un grupo de pacientes hipertensos chilenos usuarios de losartán como tratamiento antihipertensivo y comparar los genotipos encontrados con la población normal chilena, además de relacionar los diferentes polimorfismos con la dosis y posología de losartán.

\section{Pacientes y Métodos}

Tras aprobación del Comité Ético Científico de la Facultad de Medicina de la Pontificia Universidad Católica de Chile y, firma de consentimiento informado, se reclutaron 30 pacientes hipertensos usuarios de losartán atendidos en el Centro Médico San Joaquín de la Red de Salud UC-CHRISTUS. Para reducir al mínimo otros factores que podrían influir en dosis y posología del fármaco, se excluyó a todos los pacientes con patologías y medicación que pudiera modificar la respuesta enzimática 
de CYP2C9, además de aquellos con obesidad mórbida por posibles cambios en la distribución del fármaco. De esta forma se logró un grupo homogéneo de pacientes para estudio. Los criterios de inclusión y exclusión se detallan en la Tabla 1. Para realizar comparaciones de variantes alélicas y genotípicas entre hipertensos y sanos, se utilizó una base de datos del Laboratorio de Biología Molecular UC con 202 voluntarios sanos que tenían genotipificación del gen CYP2C9.

\section{Genotipificación}

En cada paciente, se obtuvo muestra de sangre venosa periférica y se extrajo ADN según el método de Lahiri y Nuremberg ${ }^{28}$. La identificación de genotipos y amplificación de variantes $C Y P 2 C 9^{*} 2$ y $C Y P 2 C 9^{\star} 3$ se realizó a través de reacción en cadena de polimerasa (PCR) y se utilizaron partidores descritos previamente en la literatura ${ }^{29}$. El análisis posterior se hizo por electroforesis capilar con detección fluorescente (ABI PRISM 3130, Applied Biosystems Carslbad, Ca, EE. UU.). Con el software Sequencing Analysis ${ }^{\circledR} 5.2$ se realizó el procesamiento automático, obteniéndose el electroferograma de la muestra para su análisis.

\section{Análisis estadístico}

La frecuencia genotípica se estableció por conteo directo de individuos que poseen cierto genotipo dividido por el número total de individuos genotipificados. Las frecuencias alélicas se establecieron por conteo directo del doble de individuos homocigotos para el alelo, más el número de individuos heterocigotos. Ese valor se dividió luego por el doble del total de individuos (número total de cromosomas).

La significancia estadística de las diferencias entre las distintas poblaciones se estableció mediante una prueba Chi-Cuadrado de Pearson o test exacto de Fisher cuando las frecuencias fueron menores al 5\%. El software utilizado para examinar la existencia de diferencias en las frecuencias alélicas entre pacientes y población general fue Epi Info ${ }^{\mathrm{TM}}$ 3.5.2.

Se calculó una razón de probabilidades (Odds Ratio, OR) que se utilizó como indicador de que un paciente pudiera requerir mayores dosis de losartán o usarlo más frecuentemente en presencia de un alelo específico (alelo ${ }^{\star} 3$ ).

\section{Resultados}

El promedio de edad fue 51,4 años con una desviación estándar (DE) de 9,6 y el índice de masa corporal (IMC) promedio fue de $29 \mathrm{k} / \mathrm{m}^{2}$. Los pacientes fueron mayoritariamente de sexo femenino $(70 \%)$. La creatinina sérica (Scr) promedio fue de $0,78 \mathrm{mg} / \mathrm{dL}$ y todos los pacientes tenían un promedio de PA < 140/90 mmHg. Sólo 10\% usaba losartán dos veces al día (b.i.d) y la mayoría (90\%) lo usaba 1 vez al día (q.d). La mayoría usaba una dosis de $50 \mathrm{mg} /$ día (80\%), 13,3\% usaba $100 \mathrm{mg} /$ día y sólo 6,7\% usaba 25 mg/día (Tabla 2).

Tabla 1. Criterios de Inclusión y Exclusión

\begin{tabular}{|ll|}
\hline Criterios de Inclusión & Criterios de Exclusión \\
\hline HTA esencial & HTA secundaria \\
\hline $\mathrm{IMC}<35$ & Obesidad con IMC $>35$ \\
\hline VFGe por MDRD $>45 \mathrm{~mL} / \mathrm{min}$ & Diabetes Mellitus \\
\hline Función hepática normal & VGFe por MDRD $<45 \mathrm{ml} / \mathrm{min}$ \\
\hline Losartán como parte del tratamiento & Insuficiencia hepática clínica \\
\hline & Consumo problemático de OH* \\
& Trastorno psiquiátrico severo** \\
\hline
\end{tabular}

HTA: Hipertensión arterial; IMC: Índice de masa corporal; VFGe: Velocidad de filtración glomerular estimada; MDRD: Modification of Diet in Renal Disease. *Edwards G et al. Alcohol-related disabilities. Geneva, World Health Organization, 1977 (WHO Offset Publication, N32). ${ }^{* *}$ Con diagnóstico por psiquiatra o en tratamiento. 
Tabla 2. Estadística descriptiva de los pacientes enrolados en este estudio

\begin{tabular}{|lc|}
\hline Edad (años) promedio (DE) & $51,4(9,6)$ \\
Sexo & $9(30 \%)$ \\
Masculino & $21(70 \%)$ \\
Femenino & 29 \\
Indice de Masa Corporal $\left(\mathrm{kg} / \mathrm{m}^{2}\right)$ & 0,78 \\
Creatinina plasmática (mg/dL) promedio & \\
Presión Arterial (mmHg) promedio & 131 \\
Presión arterial sistólica & 82 \\
Presión arterial diastólica & \\
Dosis de Losartán (\%) & 6,67 \\
25 mg & 80 \\
50 mg & 13,33 \\
100 mg & \\
Posología de Losartán (\%) & 90 \\
q.d & 10 \\
b.i.d & \\
\hline
\end{tabular}

q.d. significa una vez al día (del latín quaque die). b.i.d. significa dos veces al día (del latín bis in die).
Frecuencias alélicas y genotípicas

Las frecuencias alélicas y genotípicas obtenidas para el gen CYP2C9 se compararon con población sana chilena $(n=202)$. Se observó una diferencia significativa en la presencia del alelo ${ }^{\star} 3$, siendo mayor en la población hipertensa usuaria de losartán $(\mathrm{p}=0,041)$ y lo mismo ocurrió en la presencia del genotipo ${ }^{\star} 1 /{ }^{\star} 3$ en los sujetos hipertensos $(\mathrm{p}=0,04)$. No se observó diferencias significativas para el resto de los alelo y genotipos (Tablas 3 y 4 ).

Entre los pacientes estudiados no se observó homocigotos para los alelos ${ }^{\star} 2 \mathrm{ni}^{\star} 3$, mientras que en la población general se observó $0,5 \%$ de homocigotos para la variante ${ }^{\star} 2$. Para los heterocigotos compuestos $\left({ }^{\star} 2{ }^{\star} 3\right)$ las frecuencias genotípicas en los pacientes fueron de $3,3 \%$, mientras que la población general es de $2 \%$.

\section{Asociación de polimorfismos en el gen CYP2C9y uso de losartán}

La mayoría de los pacientes, para lograr adecuado control tensional, se encontraba usando losartán $50 \mathrm{mg}(80 \%)$ en una sola toma al día $(90 \%)$. Todos los pacientes con alelo ${ }^{\star} 2$ usaban losartán en dosis 100 mg sólo una vez al día.

Tabla 3. Frecuencias alélicas del gen CYP2C9 en población general y pacientes en tratamiento con Losartán

\begin{tabular}{|c|c|c|c|}
\hline & Alelo *1 (frecuencia) & Alelo *2 (frecuencia) & Alelo *3 (frecuencia) \\
\hline Población general $(n=202$, Alelos $=404)$ & $350 / 404 \quad(0,866)$ & $39 / 404 \quad(0,096)$ & $15 / 404 \quad(0,037)$ \\
\hline Pacientes HTA $(n=30$, Alelos $=60)$ & $51 / 60 \quad(0,85)$ & $3 / 60 \quad(0,05)$ & $(0,1)$ \\
\hline$\left(\chi^{2}\right)$ P value & 0,73 & 0,241 & 0,029 \\
\hline (Fisher) $\mathrm{P}$ value & & & 0,041 \\
\hline
\end{tabular}

Tabla 4. Frecuencias genotípicas del gen CYP2C9 en población general y pacientes en tratamiento con Losartán

\begin{tabular}{|c|c|c|c|c|c|c|}
\hline & *1/*1 (\%) & *1/*2 (\%) & *1/*3 (\%) & *2/*2 (\%) & *2/*3 (\%) & *3/*3 (\%) \\
\hline $\begin{array}{l}\text { Población general } \\
(\mathrm{n}=202)\end{array}$ & $\begin{array}{c}153 / 202 \\
(75,7)\end{array}$ & $33 / 202(16,3)$ & $\begin{array}{c}11 / 202 \\
(5,5)\end{array}$ & $\begin{array}{c}1 / 202 \\
(0,5)\end{array}$ & $\begin{array}{c}4 / 202 \\
(2)\end{array}$ & $\begin{array}{l}0 / 202 \\
(0)\end{array}$ \\
\hline $\begin{array}{l}\text { Pacientes HTA } \\
(\mathrm{n}=30)\end{array}$ & $\begin{array}{l}22 / 30 \\
(73,3)\end{array}$ & $\begin{array}{l}2 / 30 \\
(6,7)\end{array}$ & $\begin{array}{c}5 / 30 \\
(16,7)\end{array}$ & $\begin{array}{l}0 / 30 \\
(0)\end{array}$ & $\begin{array}{l}1 / 30 \\
(3,3)\end{array}$ & $\begin{array}{l}0 / 30 \\
(0)\end{array}$ \\
\hline$\left(\chi^{2}\right)$ P value & 0,775 & 0,167 & 0,024 & 0,699 & 0,634 & - \\
\hline (Fisher) P value & & 0,271 & 0,040 & 1,000 & 0,503 & - \\
\hline
\end{tabular}


Tabla 5. Riesgo de requerir mayor dosis de losartán según alelo

\begin{tabular}{|lcccc|}
\hline & Alelo *3 (\%) & Alelo *1 (\%) & OR & IC \\
\hline Losartán $100 \mathrm{mg}$ & $4 / 60(6,7)$ & $41 / 60(68,3)$ & 1,46 & $(0,01-18,64)$ \\
\hline Losartán $50 \mathrm{mg}$ & $1 / 60(1,7)$ & $7 / 60(11,7)$ & 1 & - \\
\hline
\end{tabular}

Tabla 6. Riesgo de requerir mayor frecuencia de uso de losartán según alelo

\begin{tabular}{|ccccc|}
\hline & Alelo *3 (\%) & Alelo *1 (\%) & OR & IC \\
\hline b.i.d. & $4 / 60(6,7)$ & $47 / 60(78,3)$ & 5,88 & $(0.54-62.14)$ \\
\hline q.d. & $2 / 60(3,3)$ & $4 / 60(6,7)$ & 1 & - \\
\hline
\end{tabular}

q.d. significa una vez al día (del latin quaque die). b.i.d. significa dos veces al día (del latín bis in die).

Al comparar la presencia de un alelo ${ }^{\star} 3$ versus alelo ${ }^{\star} 1$ con las dosis habituales de losartán $50 \mathrm{mg}$ y $100 \mathrm{mg}$, se determinó un OR de 1,46 (IC 0,01$18,64)$, lo que confiere mayor riesgo a la presencia de un alelo ${ }^{\star} 3$ de requerir mayor dosis de losartán (Tabla 5). Así mismo, se comparó la presencia de alelo ${ }^{*} 3$ versus alelo ${ }^{\star} 1$ en relación con la mayor frecuencia de uso de losartán y se obtuvo un OR de 5,88 (IC 0,54-62,14), lo que confiere mayor riesgo de requerir más frecuentemente el fármaco (b.i.d.) en pacientes con presencia de alelo ${ }^{\star} 3$ (Tabla 6 ).

\section{Discusión}

La hipertensión arterial es una de las causas más importante de morbilidad y mortalidad en el mundo entero, pero sólo $53 \%$ de los pacientes hipertensos con tratamiento médico logra un buen control tensional ${ }^{30}$. Dentro de los fármacos que ha mostrado más beneficios en el manejo de la hipertensión esta losartán que fue aprobado por la FDA hace ya 20 años. Durante ese tiempo, investigadores habían desarrollado una completa base de conocimiento acerca de sus beneficios ${ }^{31}$. Muchos de éstos representan un efecto de clase que es compartido por el resto de los bloqueadores de AT1, mas losartán tiene propiedades que son únicas. Por ejemplo, tiene un efecto uricosúrico ${ }^{32}$ y además atenúa la agregación plaquetaria ${ }^{33}$, lo cual no se ve con el uso de otros bloqueadores de AT1. Esto le confiere beneficios sobre el resto de los fármacos de su clase y contribuye a su amplio uso actualmente. En Chile la hipertensión arterial tiene una prevalencia de $27,6 \%{ }^{34}$ y losartán constituye un fármaco de gran uso en esta población, ya que está cubierto por el sistema de salud, tanto público como privado. Sin embargo, hasta ahora no existían estudios locales de asociación entre las variantes genéticas más frecuentes del gen CYP2C9 y las dosis y posología de losartán. Nuestra investigación permite crear una hipótesis y factibilidad de estudio para la gran cantidad de pacientes usuarios de losartán.

Al comparar las frecuencias alélicas de los pacientes hipertensos usuarios de losartán con la población general chilena, se observó datos similares para los alelos ${ }^{\star} 1 y^{\star} 2$ en el gen CYP2C9. Sin embargo, se observó significativamente una mayor frecuencia del alelo ${ }^{\star} 3$ en la población hipertensa. De la misma forma, al comparar las frecuencias genotípicas de los pacientes con la población general, se observó un mayor número de heterocigotos ${ }^{\star} 1{ }^{\star} 3$ en el gen CYP2C9 (Tablas 3 y 4 ). En análisis previos donde se caracterizó la presencia de polimorfismos de CYP2C9 en paciente usuarios de warfarina, también se demostró una tendencia en estos pacientes a ser portadores del alelo ${ }^{\star} 3$ al compararlos con personas sanas dentro del mismo grupo poblacional ${ }^{35}$. Esta tendencia podría estar dada por una asociación de estos polimorfismos con alguna condición que predispone a los pacientes a padecer enfermedades cardiovasculares y particularmente a ser hipertensos. Así como se ha demostrado que CYP2C9 participa en la homeostasis del endotelio ${ }^{36}$, también se ha observado 
que polimorfismos en CYP2C9 se relacionan con enfermedades cardiovasculares ${ }^{37}$.

Dada la baja frecuencia del alelo ${ }^{*} 2$ en la población de estudio, no fue posible determinar una asociación entre este alelo y la dosis o posología de losartán, no obstante, se observa una tendencia a requerir mayor dosis. Los resultados de investigaciones previas en poblaciones de otras partes del mundo han demostrado el impacto de las variantes ${ }^{\star} 2$ y especialmente ${ }^{\star} 3$ del gen CYP2C 9 y una actividad enzimática reducida. Por lo tanto, es necesario un mayor número de pacientes para poder evaluar correctamente si esta variante puede influir sobre la dosis y posología de losartán.

En el caso del alelo ${ }^{\star} 3$, que también presenta una baja frecuencia en la población, si fue posible encontrar una asociación con la necesidad de usar mayor dosis de losartán y una frecuencia de uso de 2 veces al día, fenómeno que podría ser explicado debido a que el alelo ${ }^{*} 3$ confiere una menor actividad enzimática de CYP2C9. Existen estudios que replican esto, demostrando que ésta última variante se asocia a menor respuesta antihipertensiva al losartán ${ }^{38} \mathrm{e}$ incluso menor respuesta antiproteinúrica en pacientes con enfermedad renal crónica ${ }^{39}$. Nuestro estudio no consideró a la proteinuria como variable, pero será de interés incluirla en un próximo estudio con mayor número de pacientes.

Hoy en día, se reconoce cada vez más la importancia del estudio fármaco-genético para establecer la dosis de distintos fármacos, sobre todo en las enfermedades cardiovasculares, como por ejemplo llegando incluso a recomendarse su análisis para ajustes en el uso de ciertos beta-bloqueadores en el caso de polimorfismos de CYP2D $6^{39}$ y particularmente en el caso polimorfismos de CYP2C9 y VKORC1 en pacientes que usan warfarina ${ }^{35}$.

Por el momento no existe una recomendación de tamizaje genético en todos los pacientes hipertensos, ya que no existe suficiente evidencia para establecer que las variantes genéticas por sí solas tengan impacto en la respuesta a la terapia. Sin embargo, demostrar que las variantes de CYP2C9 son un factor de riesgo para presentar hipertensión o que podrían predecir la dosis o posología de un fármaco tan usado y útil como losartán, permitiría ajustar de forma más precisa el tratamiento y acercarse más al adecuado control tensional, lo que se traduciría en reducción de la morbimortalidad en estos pacientes.
Este es el primer estudio fármaco-genético de losartán en población hipertensa chilena. La mayor presencia de la variante ${ }^{\star} 3$ y del genotipo ${ }^{\star} 1 /{ }^{\star} 3$ podría sugerir alguna relación entre este polimorfismo del gen CYP2C9 y la patogenia de la hipertensión. Los resultados indican una fuerte asociación entre la variante ${ }^{\star} 3$ del gen CYP2C9 que le confiere mayor riesgo al paciente portador de necesitar mayor dosis de losartán o necesidad de usarlo 2 veces al día. El siguiente paso es realizar una medición del nivel plasmático de losartán y de su metabolito E-3174 en un mayor número de pacientes con distinto genotipo y verificar el real impacto de estos polimorfismos y el metabolismo de la droga.

Finalmente, los resultados de este estudio presentan un base para diseñar un estudio más ambicioso de tamizaje en población hipertensa usuaria de losartán y su correlación con las distintas variables clínicas analizadas habitualmente en población hipertensa con buen y mal control tensional para determinar si existe un impacto clínico de dichas variantes genéticas.

\section{Referencias}

1. Weber MA. Clinical experience with the angiotensin II receptor antagonist losartan. A preliminary report. Am J Hypertens 1992; 5 (12 Pt 2): p. 247S-251S.

2. Dahlöf B, Devereux RB, Kjeldsen SE, Julius S, Beevers G, de Faire U, et al. Cardiovascular morbidity and mortality in the Losartan Intervention For Endpoint reduction in hypertension study (LIFE): a randomised trial against atenolol. Lancet 2002; 359 (9311): 995-1003.

3. James PA, Oparil S, Carter BL, Cushman WC, Dennison-Himmelfarb C, Handler J, et al. 2014 evidence-based guideline for the management of high blood pressure in adults: report from the panel members appointed to the Eighth Joint National Committee (JNC 8). JAMA 2014; 311 (5): 507-20.

4. Brunton L, Chabner B, Knollman B. Goodman and Gilman's The Pharmacological Basis of Therapeutics. 12 edition ed. 2010, New York: McGraw-Hill Professional.

5. Schwarz UI. Clinical relevance of genetic polymorphis$\mathrm{ms}$ in the human CYP2C9 gene. Eur J Clin Invest 2003; 33 Suppl 2: 23-30.

6. Stearns RA, Chakravarty PK, Chen R, Chiu SH. Biotransformation of losartan to its active carboxylic acid metabolite in human liver microsomes. Role of cytochrome P4502C and 3A subfamily members. Drug Metab Dispos 1995; 23 (2): 207-15. 
7. Yun CH, Lee HS, Lee H, Rho JK, Jeong HG, Guengerich FP. Oxidation of the angiotensin II receptor antagonist losartan (DuP 753) in human liver microsomes. Role of cytochrome P4503A(4) in formation of the active metabolite EXP3174. Drug Metab Dispos 1995; 23 (2): 285-9.

8. McCrea JB, Cribb A, Rushmore T, Osborne B, Gillen L, Lo MW, et al. Phenotypic and genotypic investigations of a healthy volunteer deficient in the conversion of losartan to its active metabolite E-3174. Clin Pharmacol Ther 1999; 65 (3): 348-52.

9. Meadowcroft AM, Williamson KM, Patterson JH, Hinderliter AL, Pieper JA. The effects of fluvastatin, a CYP2C9 inhibitor, on losartan pharmacokinetics in healthy volunteers. J Clin Pharmacol 1999; 39 (4): 418-24.

10. Yasar U, Tybring G, Hidestrand M, Oscarson M, Ingelman-Sundberg M, Dahl M, et al. Role of CYP2C9 polymorphism in losartan oxidation. Drug Metab Dispos 2001; 29 (7): 1051-6.

11. Stearns RA, Miller RR, Doss GA, Chakravarty PK, Rosegay A, Gatto GJ, et al. The metabolism of DuP 753, a nonpeptide angiotensin II receptor antagonist, by rat, monkey, and human liver slices. Drug Metab Dispos 1992; 20 (2): 281-7.

12. Sica DA, Gehr TW, Ghosh S. Clinical pharmacokinetics of losartan. Clin Pharmacokinet 2005; 44 (8): 797-814.

13. Lo MW, Goldberg MR, McCrea JB, Lu H, Furtek CI, Bjornsson TD. Pharmacokinetics of losartan, an angiotensin II receptor antagonist, and its active metabolite EXP3174 in humans. Clin Pharmacol Ther 1995; 58 (6): 641-9.

14. Rettie A, Korzekwa K, Kunze K, Lawrence R, Eddy AC, Aoyama T, et al. Hydroxylation of warfarin by human cDNA-expressed cytochrome P450: a role for P4502C9 in the etiology of (S)-warfarin drug interactions. Chem Res Toxicol 1992; 5 (1): 54-9.

15. Bajpai M, Roskos LK, Shen DD, Levy RH. Roles of cytochrome P4502C9 and cytochrome P4502C19 in the stereoselective metabolism of phenytoin to its major metabolite. Drug Metab Dispos, 1996. 24(12): 1401-3.

16. Hamman MA, Thompson GA, Hall. SD, Regioselective and stereoselective metabolism of ibuprofen by human cytochrome P450 2C. Biochem Pharmacol 1997; 54 (1): 33-41.

17. Miners JO, Birkett DJ. Use of tolbutamide as a substrate probe for human hepatic cytochrome P450 2C9. Cytochrome P450 Pt B 1996; 272: 139-45.

18. Bourrié M, Meunier V, Berger Y, Fabre G. Role of cytochrome P-4502C9 in irbesartan oxidation by human liver microsomes. Drug Metab Dispos 1999; 27 (2): 288-96.
19. DeLozier T, Kissling GE, Coulter SJ, Dai. D, Foley. JF, Bradbury JA, et al. Detection of human CYP2C8, CYP2C9, and CYP2J2 in cardiovascular tissues. Drug Metab Dispos 2007; 35 (4): 682-8.

20. Campbell WB, Gebremedhin D, Pratt PF, Harder DR. Identification of epoxyeicosatrienoic acids as endothelium-derived hyperpolarizing factors. Circ Res 1996; 78: 415-23.

21. Dhanasekaran A, Al-Saghir R, López B, Zhu D, Gutterman DD, Jacobs ER, et al. Protective effects of epoxyeicosatrienoic acids on human endothelial cells from the pulmonary and coronary vasculature. Am J Physiol Heart Circ Physiol 2006; 291 (2): H517-31.

22. Stubbins MJ, Harries LW, Smith G, Tarbit MH, Wolf CR. Genetic analysis of the human cytochrome P450 CYP2C9 locus. Pharmacogenetics 1996; 6 (5): 429-39.

23. Yasar U, Forslund-Bergengren C, Tybring G, Dorado P, Llerena A, Sjöqvist F. et al. Pharmacokinetics of losartan and its metabolite E-3174 in relation to the CYP2C9 genotype. Clin Pharmacol Ther 2002; 71 (1): 89-98.

24. Lee CR, Pieper JA, Hinderliter AL, Blaisdell JA, Goldstein JA. Losartan and E3174 pharmacokinetics in cytochrome P450 $2 \mathrm{C}^{*} 1 /{ }^{\star} 1,{ }^{\star} 1 /{ }^{\star} 2$, and ${ }^{\star} 1 /{ }^{\star} 3$ individuals. Pharmacotherapy 2003; 23 (6): 720-5.

25. Yin T, Maekawa K, Kamide K, Saito Y, Hanada H, Miyashita K. Genetic variations of CYP2C9 in 724 Japanese individuals and their impact on the antihypertensive effects of losartan. Hypertension research: official journal of the Japanese Society of Hypertension, 2008.

26. Dorado P, Beltrán LJ, Machín E, Peñas-Lledó EM, Terán E, Llerena A. Losartan hydroxylation phenotype in an Ecuadorian population: influence of CYP2C9 genetic polymorphism, habits and gender. Pharmacogenomics 2012; 13 (15): 1711-7.

27. de la Sierra A, Gorostidi M, Banegas JR, Segura J, de la Cruz JJ, Ruilope LM. Nocturnal hypertension or nondipping: which is better associated with the cardiovascular risk profile? Am J Hypertens 2014; 27 (5): 680-7.

28. Lahiri DK, Nurnberger JI Jr. A rapid non-enzymatic method for the preparation of HMW DNA from blood for RFLP studies. Nucleic Acids Res 1991; 19 (19): 5444.

29. Okada Y, Nakamura K, Adachi A, Watai Y, Horiuchi R, Yamamoto K. Development of a single-tube PCR-pyrosequencing method for the simultaneous and rapid detection of four variant alleles of CYP2C9 gene polymorphism. J Clin Pharm Ther 2008; 33(2): 187-92.

30. Chobanian AV, Bakris GL, Black HR, Cushman WC, Green LA, Izzo JL Jr, et al. The Seventh Report of the Joint National Committee on Prevention, Detection, Evaluation, and Treatment of High Blood Pressure: the JNC 7 report. JAMA 2003; 289 (19): 2560-72. 
31. Ripley E, Hirsch A. Fifteen years of losartan: what have we learned about losartan that can benefit chronic kidney disease patients? Int J Nephrol Renovasc Dis 2010; 3: 93-8.

32. Liberopoulos E, Christides D, Elisaf M. Comparative effects of losartan and irbesartan on serum uric acid in hypertensive patients with hyperuricemia and gout. J Hypertens 2002; 20 (2): 347.

33. Chłopicki S, Koda M, Chabielska E, Buczko W, Gryglewski RJ. Antiplatelet action of losartan involves TXA2 receptor antagonism but not TXA2 synthase inhibition. J Physiol Pharmacol 2000; 51 (4 Pt 1): 715-22.

34. Ministerio de Salud. Encuesta Nacional de Salud 2016-2017. https://www.minsal.cl/wp-content/ uploads/2017/11/ENS-2016-17_PRIMEROS-RESULTADOS.pdf (Consultada el 9 de julio de 2019).

35. Benavides F, Grossman N, Poggi H, Nieto E, Bertrán A, Araos D, et al. Efecto de las variantes de VKORC1 y CYP2C9 sobre la dosis de anticoagulantes orales en individuos chilenos. Rev Med Chile 2015; 143 (11): 1369-76.

36. Yasar U, Bennet AM, Eliasson E, Lundgren S, Wiman B,
De Faire U, et al. Allelic variants of cytochromes P450 2C modify the risk for acute myocardial infarction. Pharmacogenetics 2003; 13 (12): 715-20.

37. Spiecker M, Darius H, Hankeln T, Soufi M, Sattler AM, Schaefer JR, et al. Risk of coronary artery disease associated with polymorphism of the cytochrome P450 epoxygenase CYP2J2. Circulation 2004; 110 (15): 21326.

38. Lajer M, Tarnow L, Andersen S, Parving HH. CYP2C9 variant modifies blood pressure-lowering response to losartan in Type 1 diabetic patients with nephropathy. Diabet Med 2007; 24 (3): 323-5.

39. Joy MS, Dornbrook-Lavender K, Blaisdell J, Hilliard T, Boyette T, Hu Y, et al. CYP2C9 genotype and pharmacodynamic responses to losartan in patients with primary and secondary kidney diseases. Eur J Clin Pharmacol 2009; 65 (9): 947-53.

40. Ingelman-Sundberg M. Genetic polymorphisms of cytochrome P450 2D6 (CYP2D6): clinical consequences, evolutionary aspects and functional diversity. Pharmacogenomics 2004; 5 (1): 6-13. 